\title{
Transcranial Doppler-Guided Resuscitation during Transcatheter Aortic Valve Replacement
}

\author{
Khai Doan Nang, Michael Reardon, Alan Lumsden, Zsolt Garami \\ Department of Cardiovascular Surgery, Houston Methodist Hospital, Houston, TX, USA
}

\section{ABSTRACT}

Introduction: Transcatheter aortic valve replacement (TAVR) has become an optimal substitute treatment for subjects with severe aortic stenosis who cannot undergo open chest surgery or who have a high surgical risk. Transcranial Doppler (TCD) provides monitoring of microembolic signals and cerebral blood flow during TAVR and detects ongoing fluctuations of intracerebral hemodynamics during each phase of the procedure. Case report: We present the case of a patient who underwent TAVR, during which bilateral monitoring of the middle cerebral artery blood flow was performed with TCD. Conclusions: The effectiveness of transcranial Doppler to identify cerebral embolisms and variations in the cerebral hemodynamics is imperative in fending off complications during transcatheter aortic valve replacement.

Keywords: aortic stenosis, transcatheter aortic valve replacement, transcranial Doppler

\section{ARTICLE HISTORY}

Received: February 2, 2018

Accepted: February 25, 2018

\section{CORRESPONDENCE}

\section{Khai Doan Nang}

6565 Fannin St, Houston

77030 TX, USA

Tel: +17137903311

E-mail: doannang@gmail.com

\section{INTRODUCTION}

Herein, we present the case of a patient who underwent transcatheter aortic valve replacement (TAVR). With this case report, we highlight the usefulness and importance of transcranial Doppler (TCD) monitoring during this revolutionary and emerging minimal invasive technique in the detection and prevention of intraprocedural complications.

\section{CASE REPORT}

A 75-year-old male was presented with known severe aortic stenosis followed for 3 years at the Houston Methodist DeBakey Heart \& Vascular Center.

Approximately 6 months before presentation, the patient had progressively occurring dyspnea on exertion after walking roughly half a block (approx. 40 meters). Dur- ing physical examination, a systolic murmur was heard in the right 2nd intercostal area radiating to the carotid arteries. Blue edema was found bilaterally. The patient had a medical history consisting of hypertension, hyperlipidemia, coronary artery disease, myocardial infarction, diastolic heart failure, renal dysfunction, and intracranial hemorrhage. Several coronary artery bypass graft (CABG) surgeries, coronary stenting, and right hernia repair could be found in his surgical history. During his workup, electrocardiography (ECG), chest X-ray, echocardiogram, cardiac computed tomography (CT), carotid Doppler, and pulmonary function testing were performed. After clinical and imaging assessments, the patient was admitted for elective percutaneous aortic valve replacement. During the procedure, the patient's cerebral blood flow in the middle cerebral artery (MCA) was monitored bilaterally by TCD (Figure 1). 


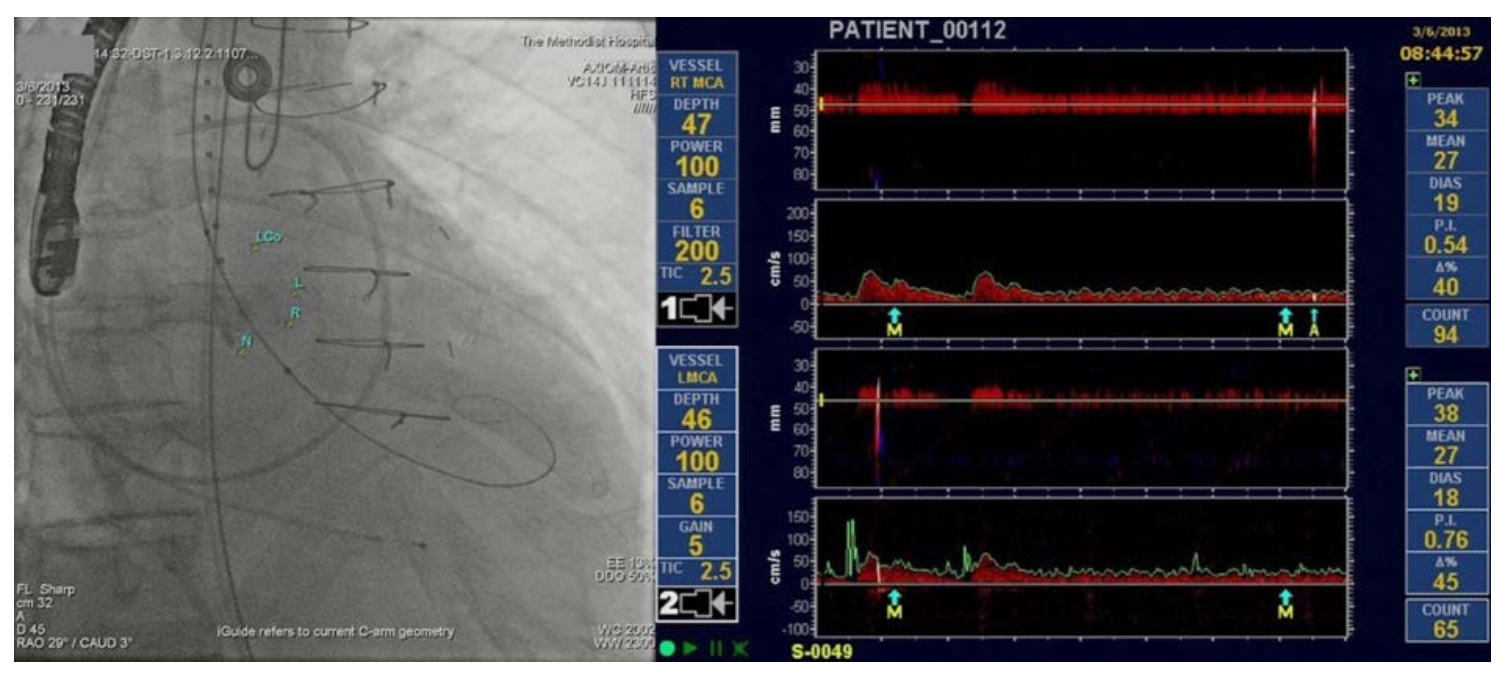

FIGURE 1. Baseline, balloon catheter positioned: a) bilateral MCA monitoring shows normal flow. b) Flow signal drop indicates the start of valvuloplasty

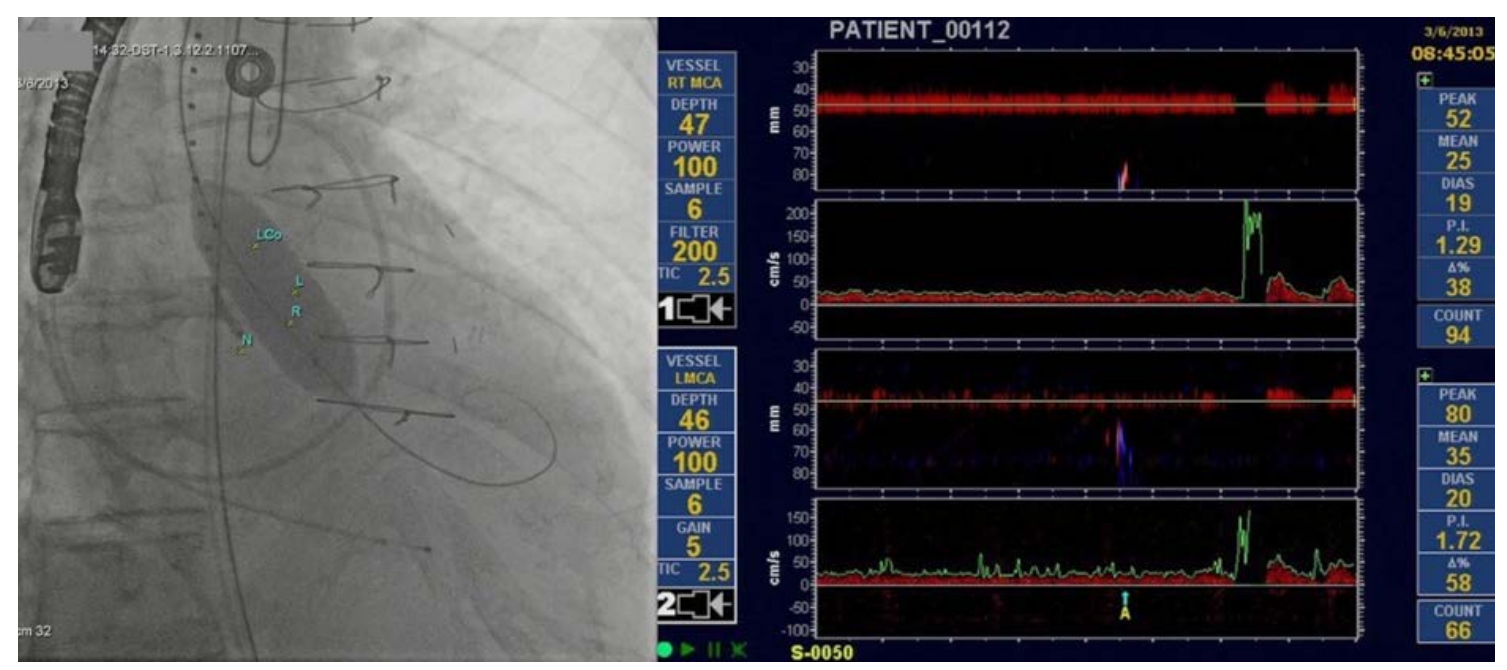

FIGURE 2. Valvuloplasty: a) minimal flow in the MCAs b) Return of pulsatile flow indicates the end of valvuloplasty
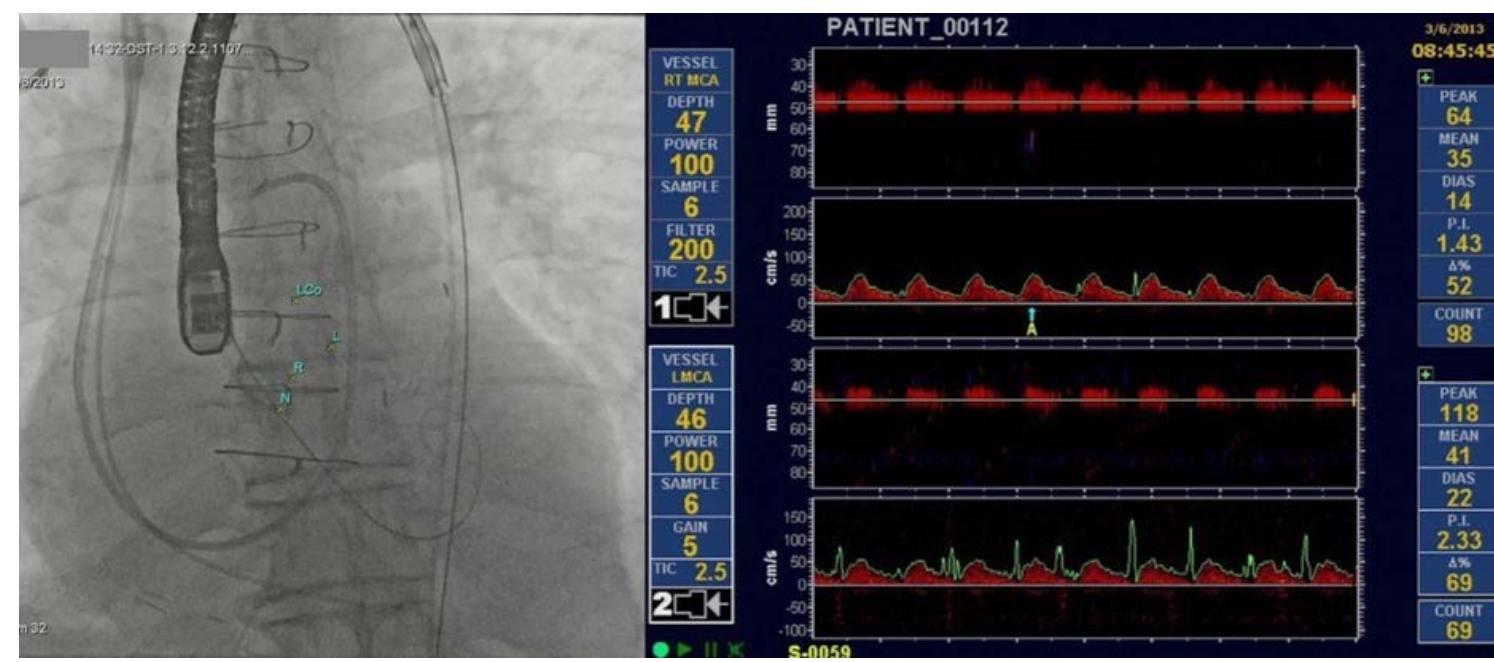

FIGURE 3. Prosthetic valve in the aortic arch: normal flow 


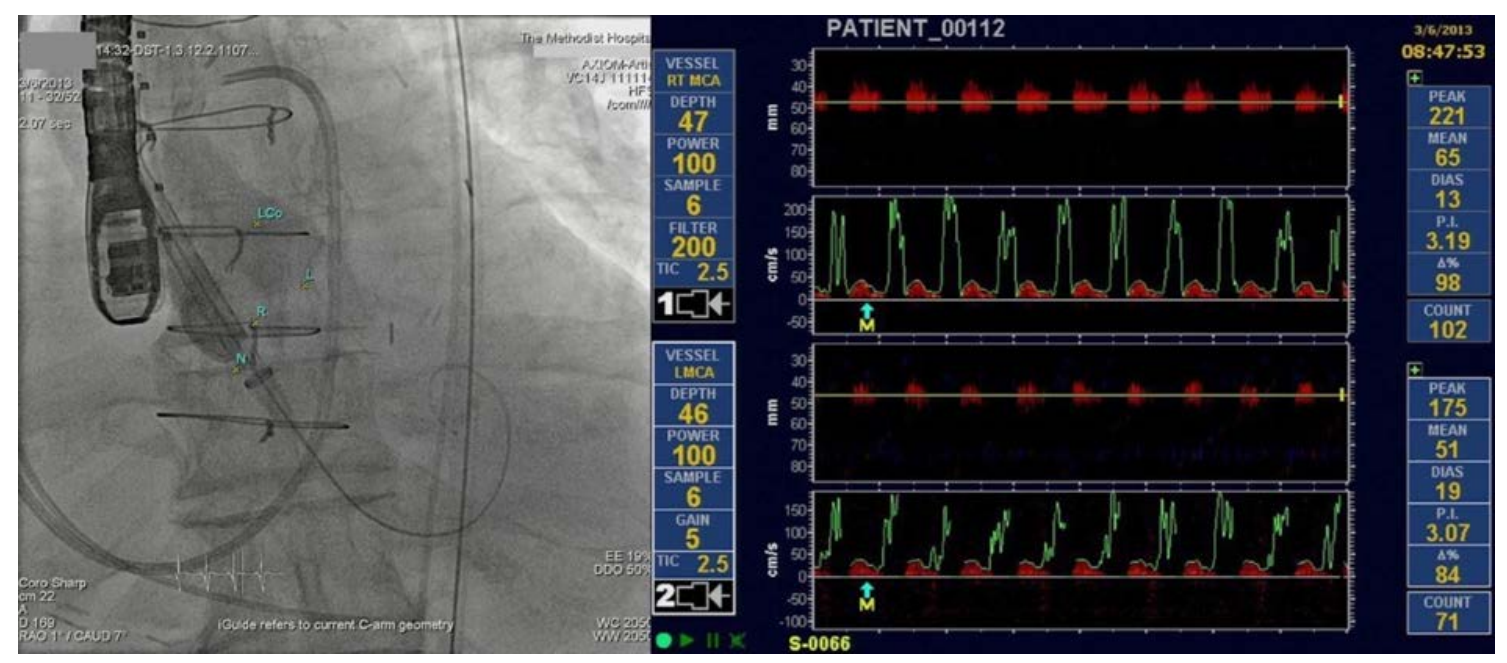

FIGURE 4. Prosthetic valve positioned in the valve: dramatically decreased MCA flow
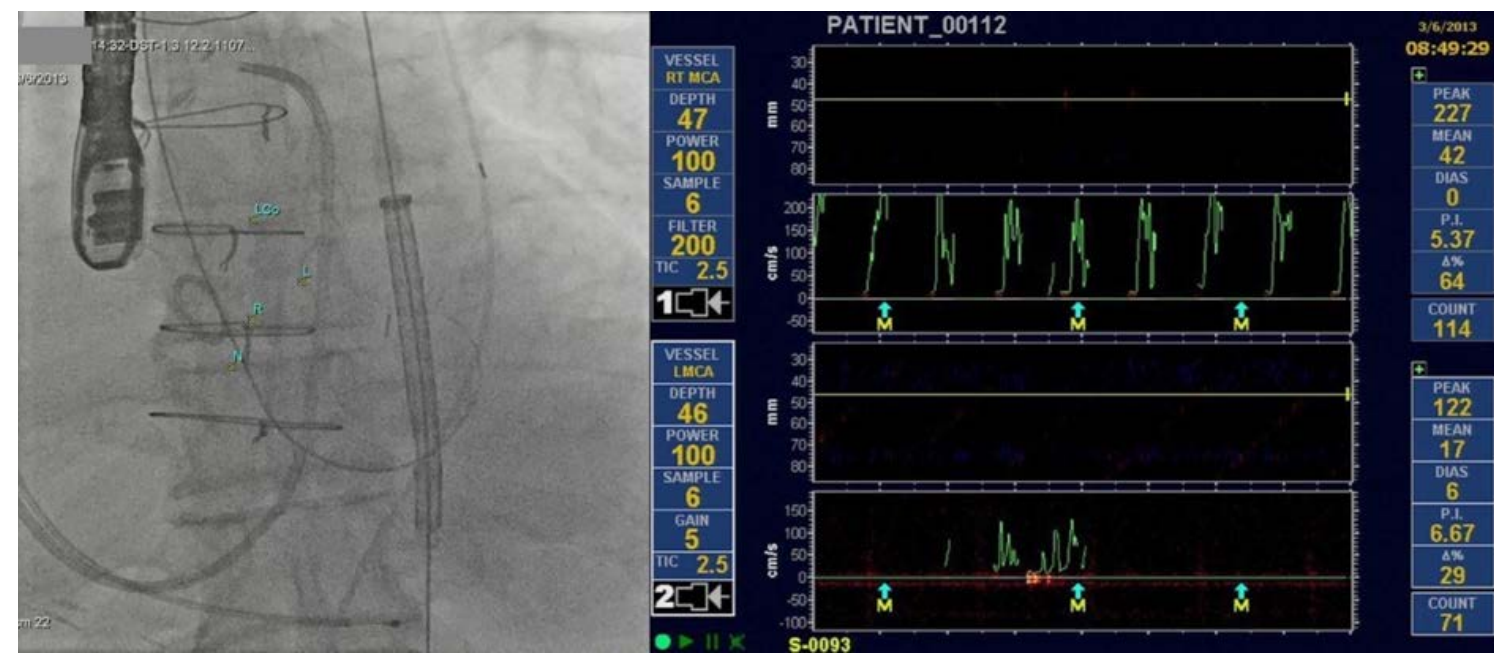

FIGURE 5. Removing prosthetic valve: bilateral signal loss, circulatory collapse

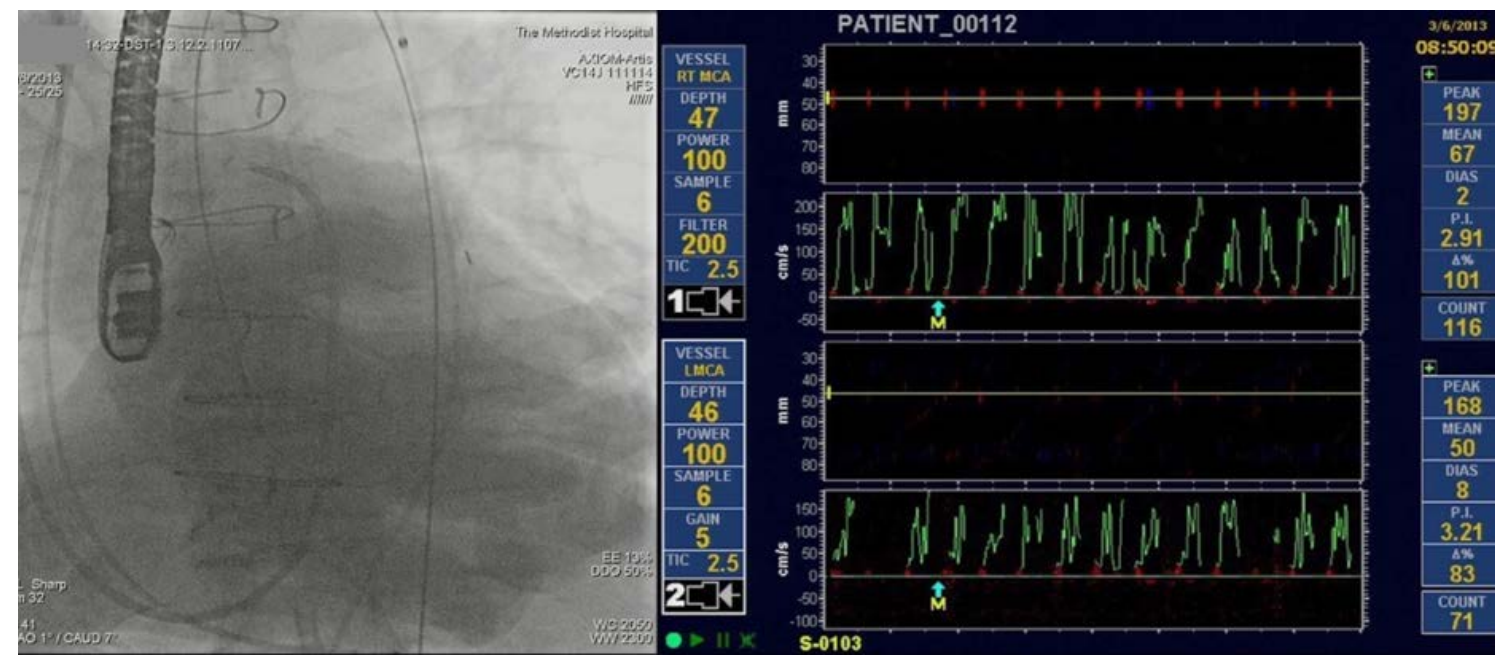

FIGURE 6. Initial CPR produced small spikes signals in the MCAs 


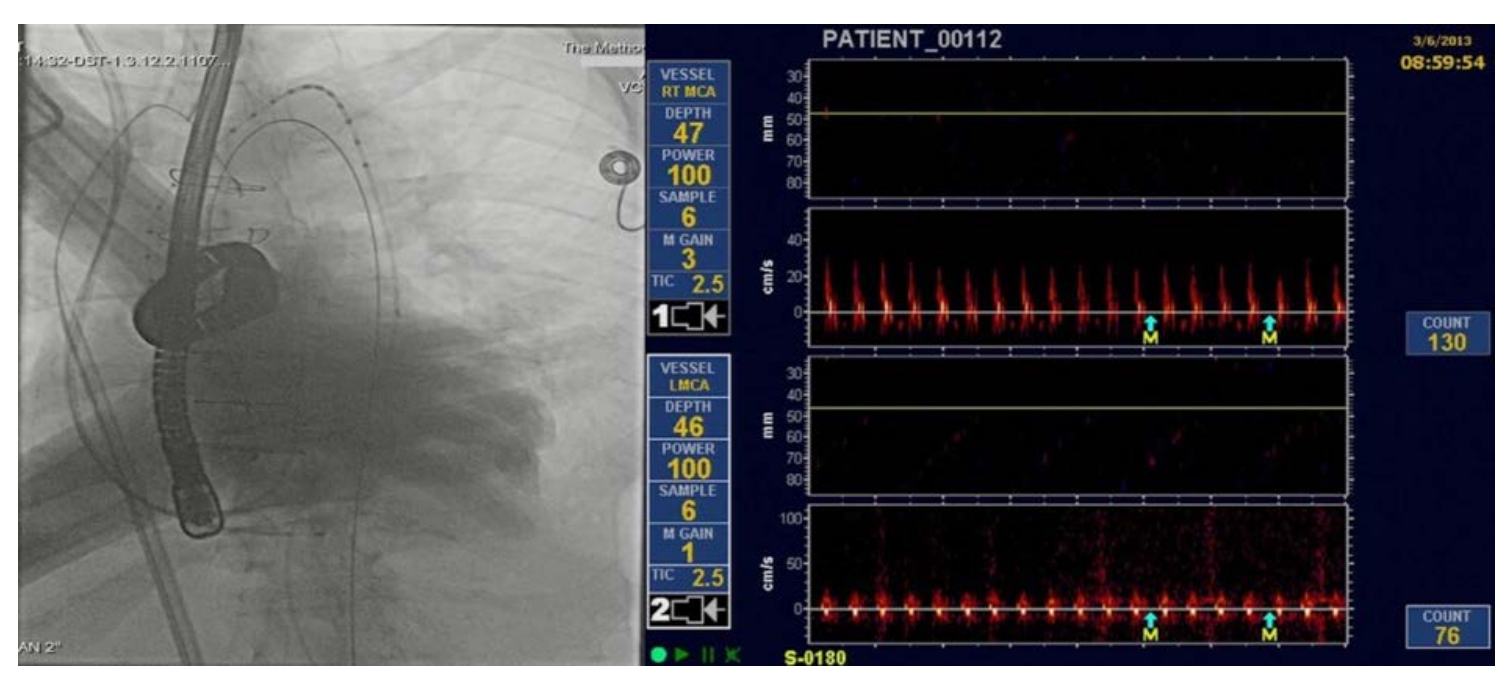

FIGURE 7. After the senior surgeon continued the CPR more noticeable spikes signals recorded

Valvuloplasty was performed (with 8 Fr Fiber Optic IAB balloon catheter by Maquet) before introducing the valve delivery system $\left(29 \mathrm{~mm}^{-}\right.$CoreValve System ${ }^{\odot}$ by Medtronic) (Figure 2). The procedure was uneventful until the prosthetic valve approached the aortic arch and valve (Figure 3). At this time, the TCD monitor recorded dramatically decreased blood flow velocities in the MCAs bilaterally (Figure 4). Blood pressure and pulse further indicated circulatory collapse, and the team immediately started cardiopulmonary resuscitation (CPR) after removing the prosthetic valve (Figure 5). First, inadequate chest compression was performed. During that period, the TCD showed small spikes in the MCAs (Figure 6). After the senior attending physician took over CPR, blood flow returned to the cerebral arteries with higher spikes in the

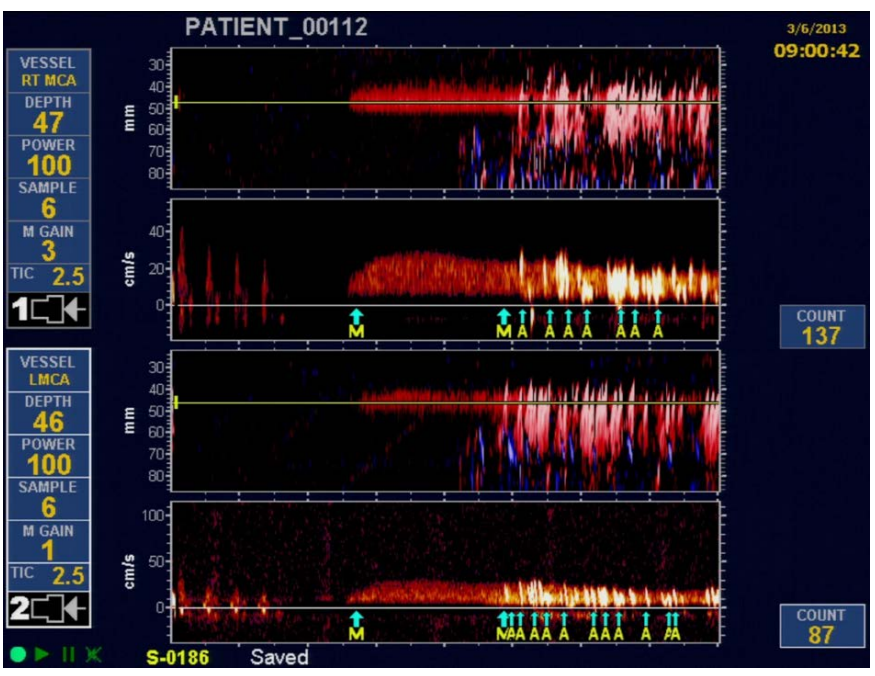

FIGURE 8. CPB initiated (from first " $M$ ") with emboli shower (" $A$ " signs on $T C D$ )
TCD, but still not comparable to baseline values (Figure 7). Via groin access, cardiopulmonary bypass (CPB) was installed, with a pump flow setting of $3.5 \mathrm{~L} / \mathrm{min}$. Highintensity transient signals (HITS) and an emboli shower were recorded (Figure 8). CPR was suspended, and after a quick check on the cannulation and connection sites, $\mathrm{CPB}$ was restarted. ECG and coronary angiography suggested intraprocedural acute inferior ST-elevation myocardial infarction secondary to right coronary artery (RCA) embolization. The occlusion in the RCA was treated with two Promus stents. TCD continued to monitor the effectiveness of reanimation and compensation of the circulatory system and showed non-pulsatile flow during CPB. After removing $\mathrm{CPB}$, improved normal pulsatile flow confirmed stabilized hemodynamics (Figure 9). The team continued

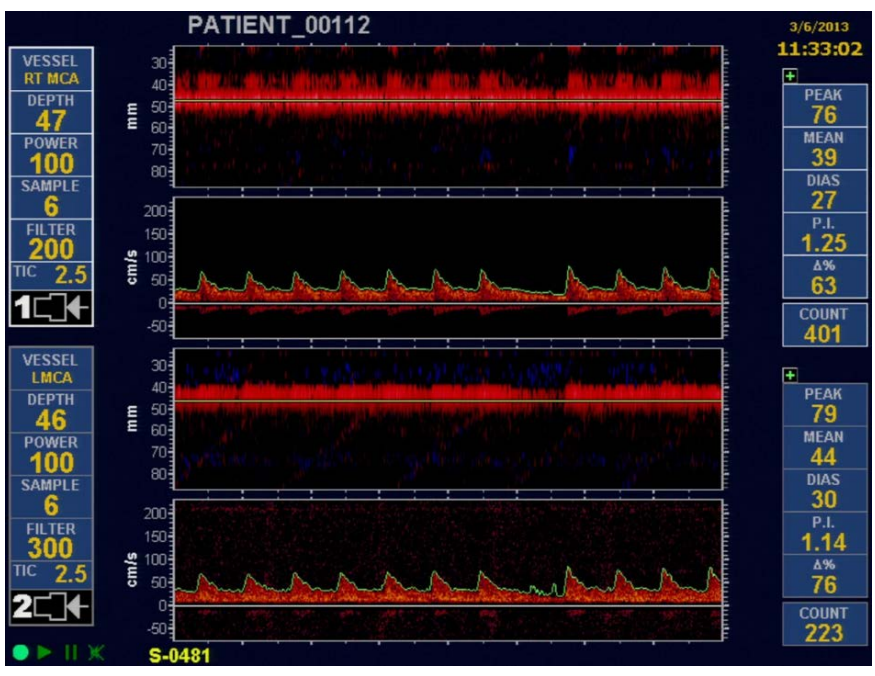

FIGURE 9. Pulsatile flow after removing CBP indicates stabilized hemodynamics 
with deployment of the valve without any further complications. After the operation, the patient was observed in the intensive care unit and discharged 6 days after the procedure. After 4 years of regular follow-ups, the patient is doing well without any cardiac complaints.

The patient agreed with publication of his data, and approval from the institution where he had been admitted was also obtained.

\section{DISCUSSIONS}

Transcatheter aortic valve replacement is an optimal alternate treatment for subjects with severe aortic stenosis who cannot undergo open chest surgery or who have a high surgical risk. ${ }^{1}$ Recent studies have suggested that this minimal invasive procedure can be beneficial in medium risk patients as well. ${ }^{2}$ The number of TAVR procedures has risen rapidly over the years and this trend is continuing. ${ }^{3}$

TCD is a noninvasive ultrasonic technique that measures velocity, direction, and properties of local blood flow in the intracranial arteries. ${ }^{4}$ It is the only technique that can be used to detect real-time perioperative cerebral flow and microemboli. Due to its repeatability and noninvasive character, TCD is currently the preferred method used to study cerebral hemodynamics and detection of microemboli. ${ }^{5}$ TCD provides continuous monitoring of microembolic signals (MES) and cerebral blood flow during TAVR and detects ongoing fluctuations of intracerebral hemodynamics during each phase of the procedure. TCD is also able to assess the contralateral cerebral circulations. Unilateral signal loss could indicate MCA obstruction and stroke, while bilateral signal loss is indicative of circulatory collapse. ${ }^{6}$

We want to emphasize the usefulness of TCD monitoring in the event of circulatory arrest and reanimation. Having the ability to detect inadequate effectiveness of the initial CPR enabled the team to reassess the situation. After effective CPR administration by the attending physician, flow reappeared on the TCD, confirming a proper reanimation technique. During this period, TCD monitoring was used as a guide for handling the situation. Even though this is a minimal invasive procedure, life-threatening and fatal complications may still occur. This case highlights the need for a multidisciplinary approach by well-trained medical staff, consisting of doctors and assistants of various medical specialties (cardiology, cardiovascular surgery, anesthesiology, imaging and perfusion specialties). ${ }^{7}$ Well-equipped operating rooms are also essential to further improve mortality rates. ${ }^{8}$

A decline in the neurocognitive function and cerebral vascular events are also potential complications of TAVR.
The reported stroke rates during the procedure range between 2 to $10 \% .{ }^{9}$ Along with symptomatic thromboembolic episodes, silent subclinical cerebroembolism occurs at an even higher degree..$^{10}$ Data suggest that cerebral hypoperfusion, hyperperfusion, and microembolization can occur at any point during the operative stage. Emboli emerging from the aortic arch, aortic valve, and the heart may vary in size, from fragments that are visible to the naked eye to 4-mm debris. ${ }^{11,12}$

\section{CONCLUSIONS}

The effectiveness of transcranial Doppler to identify cerebral embolisms and variations in the cerebral hemodynamics is imperative in fending off complications during transcatheter aortic valve replacement. We recommend further use of intraprocedural TCD monitoring during TAVR to reduce undesirable outcomes and improve patient safety.

\section{CONFLICT OF INTEREST}

Nothing to declare.

\section{REFERENCES}

1. Deeb GM, Reardon MJ, Chetcuti S, et al. 3-Year Outcomes in High-Risk Patients Who Underwent Surgical or Transcatheter Aortic Valve Replacement. J Am Coll Cardiol. 2016;67:25652574. doi: 10.1016/j.jacc.2016.03.506.

2. Svensson LG. Transcatheter Aortic Valve Replacement in Moderate-risk Aortic Stenosis Patients. Interventional Cardiology Review. 2014;9:41-43. doi: http://dx.doi. org/10.15420/icr.2011.9.1.41.

3. Reinöhl J, Kaier K, Reinecke H, et al. Effect of Availability of Transcatheter Aortic-Valve Replacement on Clinical Practice. N Engl J Med. 2015;373:2438-2447. doi: 10.1056/ NEJMoa1500893.

4. Aaslid R, Markwalder TM, Nornes H. Noninvasive transcranial Doppler ultrasound recording of flow velocity in basal cerebral arteries. J Neurosurg. 1982;57:769-774. doi: 10.3171/ jns.1982.57.6.0769.

5. Ringelstein EB, Droste DW, Babikian VL, et al. Consensus on microembolus detection by TCD. International Consensus Group on Microembolus Detection. Stroke. 1998;29:725-729.

6. Camerlingo M, Casto L, Censori B, Ferraro B, Gazzaniga GC, Mamoli A. Transcranial Doppler in acute ischemic stroke of the middle cerebral artery territories. Acta Neurol Scand. 1993;88:108-111.

7. Roselli EE, Idrees J, Mick S, et al. Emergency use of cardiopulmonary bypass in complicated transcatheter aortic valve replacement: Importance of a heart team approach. J Thorac Cardiovasc Surg. 2014;148:1413-1416. doi: 10.1016/j. jtcvs.2013.12.052. 
8. Kleiman N. Room Considerations with TAVR. Methodist Debakey Cardiovasc J. 2012;8:19-21.

9. Hecker F, Arsalan M, Walther T, et al. Managing stroke during transcatheter aortic valve replacement. Interventional Cardiology Review. 2017;12:25-30. doi: .15420/icr.2016:26:1.

10. Kahlert P, Al-Rashid F, Döttger P, et al. Cerebral Embolization During Transcatheter Aortic Valve Implantation. Circulation. 2012;126:1245-1255. doi: 10.1161/ CIRCULATIONAHA.112.092544.
11. Szeto WY, Augoustides JG, Desai ND, et al. Cerebral embolic exposure during transfemoral and transapical transcatheter aortic valve replacement. J Card Surg. 2011;26:348-354. doi: 10.1111/j.1540-8191.2011.01265.X.

12. Schmidt $T$, Akdag $O$, Wohlmuth $P$, et al. Histological Findings and Predictors of Cerebral Debris From Transcatheter Aortic Valve Replacement: The ALSTER Experience. J Am Heart Assoc. 2016;5:e004399. doi: 10.1161/JAHA.116.004399. 